\title{
Justification of mean-field coupled modulation equations
}

\author{
Guido Schneider \\ IfAM, Universität Hannover, D-30167 Hannover, Germany
}

(MS received 6 November 1995. Revised MS received 28 March 1996)

\begin{abstract}
We are interested in reflection symmetric $(x \mapsto-x)$ evolution problems on the infinite line. In the systems which we have in mind, a trivial ground state loses stability and bifurcates into a temporally oscillating, spatial periodic pattern. A famous example of such a system is the Taylor-Couette problem in the case of strongly counter-rotating cylinders. In this paper, we consider a system of coupled Kuramoto-Shivashinsky equations as a model problem for such a system. We are interested in solutions which are slow modulations in time and in space of the bifurcating pattern. Multiple scaling analysis is used in the existing literature to derive mean-field coupled Ginzburg-Landau equations as approximation equations for the problem. The aim of this paper is to give exact estimates between the solutions of the coupled Kuramoto-Shivashinsky equations and the associated approximations.
\end{abstract}

\section{Introduction}

We consider a system of coupled Kuramoto-Shivashinsky equations:

$$
\begin{aligned}
& \partial_{t} u=-\left(1+\partial_{x}^{2}\right)^{2} u-\alpha_{1} \partial_{x} u-\alpha_{2} \partial_{x}^{3} u+\varepsilon^{2} u+\partial_{x}\left(u^{2}+u v+v^{2}\right), \\
& \partial_{t} u=-\left(1+\partial_{x}^{2}\right)^{2} v+\alpha_{1} \partial_{x} v+\alpha_{2} \partial_{x}^{3} v+\varepsilon^{2} v+\partial_{x}\left(u^{2}+u v+v^{2}\right),
\end{aligned}
$$

with $t \geqq 0, x \in \mathbb{R}$, and $\alpha_{1}, \alpha_{2}, u, v \in \mathbb{R}$. The variable $\varepsilon$ with $1 \gg \varepsilon^{2}>0$ is used as a bifurcation parameter. The linearisation at $(u, v) \equiv(0,0)$ possesses eigenfunctions of the form $(u, v)(x, t)=\left(a_{k} e^{i k x}, b_{k} e^{i k x}\right),\left(a_{k}, b_{k} \in \mathbb{C}, k \in \mathbb{R}\right)$ with associated eigenvalues $\hat{\lambda}_{1,2}\left(k, \varepsilon^{2}\right)=-\left(1-k^{2}\right)^{2} \mp \alpha_{1} i k \pm a_{2} i k^{3}+\varepsilon^{2}$. We observe that $\operatorname{Re} \hat{\lambda}_{1,2}\left(k, \varepsilon^{2}\right)$ is positive for wave numbers $k$ close to the values $\pm k_{c}= \pm 1$.

Centre manifold theory (cf. [9]) allows us to exploit the bifurcation scenario in the weakly unstable case if we assume spatial periodicity with period $2 \pi$ for the solutions. If we suppress the spatial periodicity assumption, a whole band of Fourier modes with positive growth rates appears. Thus, centre manifold theory is no longer available to describe the dynamics of the bifurcating solutions. We expect that the bifurcating solutions are slow modulations in time and in space of the bifurcating pattern $e^{i x}$ due to the form of $\hat{\lambda}_{1,2}$. Therefore, we make the ansatz.

$$
\begin{aligned}
u=\psi_{u}(t, x, \varepsilon)[A, B]= & \varepsilon A\left(\varepsilon(x-\alpha t), \varepsilon^{2} t\right) e^{i(x-\beta t)}+\varepsilon^{2} \sum_{j=0, \pm 2} A_{2}^{j}\left(\varepsilon(x-\alpha t), \varepsilon^{2} t\right) e^{2 i x} \varepsilon^{i j \beta t} \\
& \left.+\varepsilon^{2} / 2 A_{0}^{0}\left(\varepsilon(x-\alpha t), \varepsilon^{2} t\right), \varepsilon^{2} t\right)+c . c ., \\
v=\psi_{v}(t, x, \varepsilon)[A, B]= & \varepsilon B\left(\varepsilon(x+\alpha t), e^{2} t\right) e^{i(x+\beta t)}+\varepsilon^{2} \sum_{j=0, \pm 2} B_{2}^{j}\left(\varepsilon(x+\alpha t), \varepsilon^{2} t\right) e^{2 i x} e^{i j \beta t} \\
& +\varepsilon^{2} / 2 B_{0}^{0}\left(\varepsilon(x+\alpha t), \varepsilon^{2} t\right)+c . c .,
\end{aligned}
$$

with $\alpha, \beta \in \mathbb{R}$. We introduce the spatial translation operator $\left(\mathscr{T}_{y} u\right)(x, t)=u(x+y, t)$ 
and the spatial scaling operator $\left(\mathscr{S}_{\varepsilon} u\right)(x, t)=u(\varepsilon x, t)$. Inserting the ansatz (1.2) into (1.1) and equating the coefficients in front of $\varepsilon^{n} e^{i m x} e^{i j \beta t}$ to zero gives

$$
\alpha=-\alpha_{1}+3 \alpha_{2}, \quad \beta=\alpha_{1}-\alpha_{2},
$$

and the equations for $A=A(X, T), B=B(X, T), A_{m}^{j}=A_{m}^{j}(X, T)$ and $B_{m}^{j}=$ $\mathrm{B}_{m}^{j}(\mathrm{X}, \mathrm{T})$, where $T=\varepsilon^{2} t$ and $X=\varepsilon x$.

Elimination of the variables $A_{m}^{j}$ and $B_{m}^{j}$ for $m=0,2 ; j=0, \pm 2$ by the algebraic equations

$$
\begin{gathered}
\left(\mathscr{T}_{-\alpha T / \varepsilon} A_{0}^{0}\right)=\left(\mathscr{T}_{\alpha T / \varepsilon} B_{0}^{0}\right)=0, \\
\left(9-6 i \alpha_{2}\right)\left(\mathscr{T}_{-\alpha T / \varepsilon} A_{2}^{-2}\right)=\left(9-4 i \alpha_{1}+10 \alpha_{2}\right)\left(\mathscr{T}_{\alpha T / \varepsilon} B_{2}^{-2}\right)=2 i\left(\mathscr{T}_{-\alpha T / \varepsilon} A\right)^{2}, \\
\left(9+2 i \alpha_{1}-8 i \alpha_{2}\right)\left(\mathscr{T}_{-\alpha T / \varepsilon} A_{2}^{0}\right)=\left(9-2 i \alpha_{1}+8 i \alpha_{2}\right)\left(\mathscr{T}_{\alpha T / \varepsilon} B_{2}^{0}\right)=2 i\left(\mathscr{T}_{-\alpha T / \varepsilon} A\right)\left(\mathscr{T}_{\alpha T / \varepsilon} B\right), \\
\left(9+4 i \alpha_{1}-10 i \alpha_{2}\right)\left(\mathscr{T}_{-\alpha T / \varepsilon} A_{2}^{2}\right)=\left(9+6 i \alpha_{2}\right)\left(\mathscr{T}_{\alpha T / \varepsilon} B_{2}^{2}\right)=2 i\left(\mathscr{T}_{\alpha T / \varepsilon} B\right)^{2},
\end{gathered}
$$

which we have obtained by the above procedure gives finally

$$
\begin{aligned}
& \partial_{T} A=A+4 \partial_{X}^{2} A-\left(\gamma_{1} A|A|^{2}+a_{1}+a_{2}\right), \\
& \partial_{T} B=B+4 \partial_{X}^{2} B-\left(\gamma_{6} B|B|^{2}+b_{1}+b_{2}\right),
\end{aligned}
$$

where

$$
\begin{aligned}
a_{1}= & \gamma_{5} A\left(\mathscr{T}_{2 \alpha T / \varepsilon} \bar{B}\right)\left(\mathscr{T}_{2 \alpha T / \varepsilon} B\right), \\
a_{2}= & \gamma_{4} A\left(\mathscr{T}_{2 \alpha T / \varepsilon} \bar{B}\right) A e^{-2 i \beta T / \varepsilon^{2}}+\gamma_{2} A \bar{A}\left(\mathscr{T}_{2 \alpha T / \varepsilon} B\right) e^{2 i \beta T / \varepsilon^{2}} \\
& +\gamma_{3}\left(\mathscr{T}_{2 \alpha T / \varepsilon} B\right) \bar{A}\left(\mathscr{T}_{2 \alpha T / \varepsilon} B\right) e^{4 i \beta T / \varepsilon^{2}}+\gamma_{6}\left(\mathscr{T}_{2 \alpha T / \varepsilon} B\right)\left(\mathscr{T}_{2 \alpha T / \varepsilon} \bar{B}\right)\left(\mathscr{T}_{2 \alpha T / \varepsilon} B\right) e^{2 i \beta T / \varepsilon^{2}}, \\
b_{1}= & \gamma_{2} B\left(\mathscr{T}_{-2 \alpha T / \varepsilon} \bar{A}\right)\left(\mathscr{T}_{-2 \alpha T / \varepsilon} A\right), \\
b_{2}= & \gamma_{5}\left(\mathscr{T}_{-2 \alpha T / \varepsilon} A\right) \bar{B} B e^{-2 i \beta T / \varepsilon^{2}}+\gamma_{4}\left(\mathscr{T}_{-2 \alpha T / \varepsilon} A\right)\left(\mathscr{T}_{-2 \alpha T / \varepsilon} A\right) \bar{B} e^{-4 i \beta T / \varepsilon^{2}} \\
& +\gamma_{3} B\left(\mathscr{T}_{-2 \alpha T / \varepsilon} \bar{A}\right) B e^{2 i \beta T / \varepsilon^{2}}+\gamma_{1}\left(\mathscr{T}_{-2 \alpha T / \varepsilon} A\right)\left(\mathscr{T}_{-2 \alpha T / \varepsilon} \bar{A}\right)\left(\mathscr{T}_{-2 \alpha T / \varepsilon} A\right) e^{-2 i \beta T / \varepsilon^{2}} .
\end{aligned}
$$

As usual, $\bar{F}$ denotes the complex conjugate of $F$. For problem (1.1) we have the coefficients

$$
\begin{array}{ll}
\gamma_{1}=\left(\frac{4}{9-6 i \alpha_{2}}+\frac{2}{9-4 i \alpha_{1}+10 i \alpha_{2}}\right), & \gamma_{2}=\left(\frac{4}{9+2 i \alpha_{1}-8 i \alpha_{2}}+\frac{2}{9-2 i \alpha_{1}+8 i \alpha_{2}}\right), \\
\gamma_{3}=\left(\frac{4}{9+4 i \alpha_{1}-10 i \alpha_{2}}+\frac{2}{9+6 i \alpha_{2}}\right), & \gamma_{4}=\left(\frac{2}{9-6 i \alpha_{2}}+\frac{4}{9-4 i \alpha_{1}+10 i \alpha_{2}}\right), \\
\gamma_{5}=\left(\frac{2}{9+2 i \alpha_{1}-8 i \alpha_{2}}+\frac{4}{9-2 i \alpha_{1}+8 i \alpha_{2}}\right), & \gamma_{6}=\left(\frac{2}{9+4 i \alpha_{1}-10 i \alpha_{2}}+\frac{4}{9+6 i \alpha_{2}}\right) .
\end{array}
$$

Throughout the paper, we assume that $\alpha_{1}$ and $\alpha_{2}$ are given in such a way that $\alpha$ and $\beta$ are nonvanishing real constants which are independent of the bifurcation parameter $\varepsilon$. Under this assumption the equations (1.4) still depend strongly on the small bifurcation parameter $\varepsilon$. The aim of the existing literature (see below) is to obtain equations which are independent of the small parameter $\varepsilon$. We follow the arguments of these papers and neglect the terms $a_{2}$ and $b_{2}$ since they are highly 
oscillating in time for $\varepsilon \rightarrow 0$. Secondly, the terms $\bar{B} B$, and $\bar{A} A$ depend on the fast drift terms $X \pm 2 \alpha T / \varepsilon$ in $a_{1}$ and $b_{1}$. Thus, it is widely expected that the dynamics of $A$ and $B$ is only influenced by the mean value of the terms $\bar{B} B$ and $\bar{A} A$ over a certain space region. Thus, a so-called mean-field coupled system of Ginzburg-Landau equations is considered:

$$
\begin{aligned}
& \partial_{T} A=A+4 \partial_{X}^{2} A-\left(\gamma_{1} A|A|^{2}+\gamma_{5} A\left\langle\left|\mathscr{T}_{2 \alpha T / \varepsilon} B\right|^{2}\right\rangle_{L}\right), \\
& \partial_{T} B=B+4 \partial_{X}^{2} B-\left(\gamma_{6} B|B|^{2}+\gamma_{2} B\left\langle\left|\mathscr{T}_{-2 \alpha T / \varepsilon} A\right|^{2}\right\rangle_{L}\right),
\end{aligned}
$$

where

$$
\langle F\rangle_{L}(Y)=\frac{1}{2 L} \int_{-L}^{L} F(Y+X) d X
$$

for a fixed $L>0$. These equations are independent of the bifurcation parameter $\varepsilon$ if we assume $A(X)=A(X+q), B(X)=B(X+q)$ and $L=q$ for a $q>0$ fixed. In this case, the shift operator $\mathscr{T}_{2 \alpha T / \varepsilon}$ acts trivially on the terms $\left\langle\left|\mathscr{T}_{-2 \alpha T / \varepsilon} A\right|^{2}\right\rangle_{L}$ and $\left\langle\left|\mathscr{T}_{2 \alpha T / \varepsilon} B\right|^{2}\right\rangle_{L}$ since they are constants in space. It is the aim of this paper to show exact estimates between the solutions of (1.1) and the approximations which are obtained by the solutions of (1.5). In the last case our result, which is formulated in Theorem 1.2, simplifies as follows.

THEOREM 1.1. Let $\|u\|_{H_{l, u}^{n}}=\sup _{m \in \mathbb{R}}\|u\|_{H^{n}(m, m+1)}$. For arbitrary, but fixed, $q>0$ the following holds: let $(A, B) \in C\left(\left[0, T_{0}\right],\left(H_{l, u}^{8}\right)^{2}\right)$ be solutions of $(1.5)$ to $L=q$ which fulfil $A(X)=A(X+q), B(X)=B(X+q)$, and $\sup _{T \in\left[0, T_{0}\right]}\|(A, B)\|_{\left(H_{l, u}^{8}\right)^{2}} \leqq C_{1}<\infty$, for a constant $C_{1}$. Then there exists $\varepsilon_{0}, C>0$, such that for all $\varepsilon \in\left(0, \varepsilon_{0}\right)$ there are solutions $(u, v)$ of $(1.1)$ with

$$
\sup _{0 \leqq t \leqq T_{0} / \varepsilon^{2}}\left\|(u, v)-\left(\psi_{u}[A, B], \psi_{v}[A, B]\right)\right\|_{\left(H_{1, u}^{4}\right)^{2} \leqq C \varepsilon^{2}}
$$

The error of order $\mathcal{O}\left(\varepsilon^{2}\right)$ is small compared with the order $\mathcal{O}(\varepsilon)$ of the solution and of the approximation.

The question arises as to how large the length $L$ of the spatial region can be chosen such that there are really solutions of (1.1) which can be described by (1.5). We will show that solutions of the original system (1.1) exist which can be approximated by the solutions of (1.5), if $L$ is not too large.

THEOREM 1.2. For arbitrary, but fixed, $q>0$ and $v \in[0,1)$ the following holds: let $\varepsilon \in(0,1) \mapsto(A, B) \in C\left(\left[0, T_{0}\right],\left(H_{l, u}^{8}\right)^{2}\right)$ be a family of solutions of $(1.5)$ to $L=q / \varepsilon^{v}$ which fulfils $\sup _{\varepsilon \in(0,1)} \sup _{T \in\left[0, r_{0}\right]}\|(A, B)\|_{\left(H_{l, u}^{8}\right)^{2}} \leqq C_{1}<\infty$, for a constant $C_{1}$. Then there exist $\varepsilon_{0}, C>0$, such that for all $\varepsilon \in\left(0, \varepsilon_{0}\right)$ there are solutions $(u, v)$ of $(1.1)$ with

$$
\sup _{0 \leqq t \leqq T_{0} / \varepsilon^{2}}\left\|(u, v)-\left(\psi_{u}[A, B], \psi_{v}[A, B]\right)\right\|_{\left(H_{l, u}^{4}\right)^{2} \leqq C \varepsilon^{2-\nu}}
$$

Corollary 1.3. Assume a spatial period $L=q / \varepsilon^{v}$ for (1.5) with $v \in[0,1)$. Then $\langle\vec{A} A\rangle_{L}$ and $\langle\bar{B} B\rangle_{L}$ are constants in space and depend only on time. This yields to a spatial period $q / \varepsilon^{v+1}$ for the original system (1.1). In this case, the equations (1.5) are independent of $\varepsilon$. Only the underlying interval depends on $\varepsilon$.

The theory of modulation equations for dissipative systems started with the famous paper of Newell and Whitehead [16] in 1969, in which they derived a single 
Ginzburg-Landau equation for Bérnard's problem. In the last few years, approximation properties for single Ginzburg-Landau equations in the sense of Theorem 1.2 have been shown, for instance, in $[1,4,10,21,25]$.

Mean-field coupled modulation equations are derived, for instance, in $[5,6,11-14,17,18]$ for several problems. There are two justification results in the sense of Theorem 1.2. In [19] a system of coupled nonlinear Schrödinger equations is justified as an approximation system for the Sine-Gordon equation. For this problem, highly oscillating terms as in $a_{2}$ and $b_{2}$ do not appear. In [26] systems similar to (1.4) and (1.5) are compared. In both papers the case $v=0$ is considered. In contrast to these papers, our system (1.1) contains quadratic terms which are responsible for the difficulties in the justification of the Ginzburg-Landau equation. See, for instance, [20].

Here we consider a model problem, but we expect that it is possible to transfer our analysis to more general problems. System (1.1) stands as a model for a translationally invariant dissipative system in a cylindrical domain with one unbounded space direction close to the threshold of instability. At criticality, a temporally oscillating, spatial periodic pattern with period $2 \pi / k_{c}$ bifurcates from the trivial branch. Mean-field coupled Ginzburg-Landau equations can be derived if the system possesses a certain symmetry under reflections $x \mapsto-x$, the so-called reversibility. For such systems, all eigenvalues which occur for the wave number $k$ must also appear for the wave number $-k$. The eigenfunctions of such systems are of the form $e^{i k x} f_{k}(z)$, with $k \in \mathbb{R}$ and $f_{k}(z)$ a Banach-space-valued function which is defined on the bounded cross-section alone. For fixed wave number $k$, an ordered set of eigenvalues $\left(\tilde{\lambda}_{j}(k)\right)_{j \in \mathbb{N}}$ with $\operatorname{Re} \tilde{\lambda}_{j} \geqq \operatorname{Re} \tilde{\lambda}_{j+1}$ occurs. The curves $\tilde{\lambda}_{1,2}\left(k, \varepsilon^{2}\right)$ of the most unstable eigenvalues correspond to the curves $\hat{\lambda}_{1,2}\left(k, \varepsilon^{2}\right)$ in our model problem. In the general case, $\operatorname{Im} \tilde{\lambda}_{1}^{\prime}\left(k_{c}, 0\right)$ takes the role of $\alpha$ and $-\operatorname{Im} \tilde{\lambda}_{1}\left(k_{c}, 0\right)$ the role of $\beta$. A famous example of such a situation is the Taylor-Couette problem in the case of strongly counter-rotating cylinders [3]. In our model problem, the nonlinear terms do not respect the reflection symmetry described above.

We finish this introduction with the interesting observation that $\left\|\langle\bar{A} A\rangle_{s}-1 / 2\right\|_{H_{1, u}^{n}}=\mathcal{O}\left(\varepsilon^{1 / 2}\right)$ for $\varepsilon$-independent functions $A$ which vanish for $|x| \rightarrow \infty$. Thus, the equations (1.5) decouple for such $A \mathrm{~s}$ and $B \mathrm{~s}$ in lowest order. As a consequence, solutions $A$ and $B$ of (1.5) to $\varepsilon$-independent compactly supported initial conditions do not influence each other on timescales of order $\mathcal{O}\left(1 / \varepsilon^{2}\right)$. There exists a wide class of other initial conditions on the infinite line which lead to such a decoupling and to a nondependence of (1.5) on the small bifurcation parameter $\varepsilon$ in the leading order. Therefore, it is advantageous to include the case $v>0$ in our analysis and not to restrict ourselves to $v=0$. This observation is of great importance for hyperbolic problems, since the modulation equations which occur in this case (NLS-, KdV-equations) possess solitons of homoclinic form. For instance, in [24] it is shown that the Boussinesq equation decouples into two independent KdV-equations for initial conditions which decay sufficiently fast for $|x| \rightarrow \infty$. See also [19], where this decoupling is shown for the Sine-Gordon equation and the nonlinear Schrödinger equation as a modulation equation.

This paper is organised as follows. As a first step, we show that the solutions of system (1.1) can be approximated by the solutions of system (1.4). This part follows strongly along the lines of [20]. Therefore, we recommend the reading of [20] 
together with this paper. In a second step, we show that the solutions of (1.4) can be approximated by the solutions of (1.5). Moreover, we explain why the attractivity of solutions in the Ginzburg-Landau form (1.2) (cf. $[7,15,23]$ ) also holds for our system (1.1). The local existence and uniqueness for solutions of (1.4) and (1.5) is handled in Lemma 2.5 .

Throughout this paper, constants are uniformly denoted by $C$. Moreover, we assume $0<\varepsilon^{2} \ll 1$.

\section{Transfer of the classical results}

In this section, we show that solutions of (1.1) can be approximated by the solutions of (1.4). In order to do so, we follow the lines of the existing literature about the validity of the Ginzburg-Landau equation. We start this section by introducing the functional analytic set-up, followed by a remark on the attractivity of the set of solutions which are in Ginzburg-Landau form (1.2).

\section{Functional analytic set-up for the infinite line}

We recommend the reading of [15] to those interested in the details of the following functional analytic set-up. Here we only cite definitions and theorems which are necessary for what follows. We define

$$
\tilde{H}_{l, u}^{n}=\left\{u \in L_{\mathrm{loc}}^{2} \mid \partial_{x}^{j} u \in L_{\mathrm{loc}}^{2},(j=0, \ldots, n),\|u\|_{\tilde{H}_{1, u}^{n}}=\sup _{y \in \mathbb{R}}\|u\|_{\tilde{H}^{n}(y, y+1)}<\infty\right\},
$$

the space of uniform locally square-integrable Sobolev functions. Moreover, we define the Banach space

$$
H_{l, u}^{n}=\left\{u \in \tilde{H}_{l, u}^{n} \mid\left\|\mathscr{T}_{y} u-u\right\|_{H_{l, u}^{n}} \rightarrow 0 \text { for } y \rightarrow 0\right\},
$$

where $\left(\mathscr{T}_{y} u\right)(x)=u(x+y)$ again denotes the translation operator. This space is equipped with the norm of $\tilde{H}_{l, u}^{n}$. We write $L_{l, u}^{2}$ for $H_{l, u}^{0}$. The space $H_{l, u}^{n}$ is a dense subspace of $H_{l, u}^{m}$ for $m<n$, in contrast to $H_{l, u}^{n} \subset \tilde{H}_{l, u}^{m}$. As a consequence of this density, the theory of analytic semigroups can be applied in the spaces $H_{l, u}^{n}$.

In these spaces so-called multipliers can be defined. Multipliers $m$ are operators which are defined by multiplying the Fourier transform $\hat{u}=\mathscr{F} u$ of a function $u$ with an $\hat{m} \in L^{\infty}(\mathbb{R}, \mathbb{C})$ in Fourier space. In [21] it is shown that it is possible to define operators in the physical space in this way.

LEMMA 2.1. Let $q, s \geqq 0$ and $w_{s-q}(k)=\left(1+k^{2}\right)^{(s-q) / 2} \hat{m}(k) \in C_{b}^{2}(\mathbb{R}, \mathbb{C})$. Then $M: H_{l, u}^{q} \rightarrow$ $H_{l, u}^{s} ; u \mapsto \mathscr{F}^{-1}(\hat{m}, \mathscr{F} u)$ is well defined with the estimate

$$
\|M u\|_{H_{l, u}^{s}} \leqq C(q, s)\left\|w_{s-q}\right\|_{C_{b}^{2}(\mathbb{R}, \mathbb{C})}\|u\|_{H_{l, u}^{q}},
$$

where $C(q, s)$ does not depend on $\hat{m}$.

REMARK 2.2. Assume $q \geqq 1$ and $w_{s-q}(k)=\left(1+k^{2}\right)^{(s-q) / 2} \hat{m}(k) k \in C_{b}^{2}(\mathbb{R}, \mathbb{C})$. Then $M: H_{l, u}^{q} \rightarrow H_{l, u}^{s} ; u \mapsto \mathscr{F}^{-1}\left(\hat{m} \mathscr{F} \partial_{x} u\right)$ is well defined with the estimate (2.1). This is demonstrated similarly to the proof of the above Lemma 2.1 in [21].

Note that the translation operator $\mathscr{T}_{y}$, all differential operators, analytic semigroups and many more operators can be represented by multipliers. The estimate in Lemma 2.1 does not give optimal estimates for many operators, in particular 
not for the translation operator. We will denote multipliers in Fourier space with

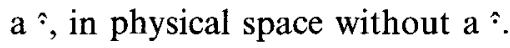

\section{The attractivity}

It is known (cf. $[2,7,8,15,23]$ ) that all small solutions of a single KuramotoShivashinsky equation develop in such a way that after a time $\mathcal{O}\left(1 / \varepsilon^{2}\right)$ they can be approximated by the solutions of the associated Ginzburg-Landau equation. In order to do so, it has to be shown that after this time the solutions can be represented in the form (2.2). It is obvious if we look at the proofs in $[2,7,23]$ that the attractivity result also holds for the coupled system (1.1). Nowhere in the proofs is it required that a scalar problem be considered or that the problem possess real eigenvalues. So we state without proof:

Theorem 2.3. For fixed $C>0, N \in \mathbb{N}$, and all initial conditions $(u(0), v(0))$ of $(1.1)$ with $\|\left(u(0), v(0) \|_{\left(H_{l, u}^{4}\right)^{2} \leqq C \varepsilon}\right.$ the following is true: there exist $\varepsilon_{0}(C, N), T_{0}(C, N)>0$ such that for all $\varepsilon \in\left(0, \varepsilon_{0}\right)$ we obtain a representation of the solution $(u, v)$ at a time $T_{0} / \varepsilon^{2}$ through

$$
\begin{aligned}
& u\left(T_{0} / \varepsilon^{2}, x, \varepsilon\right)=\sum_{m=-N}^{N} \varepsilon^{\gamma(m)} A_{m}(\varepsilon x) e^{i m x}+\mathcal{O}\left(\varepsilon^{(N+3) / 2}\right), \\
& v\left(T_{0} / \varepsilon^{2}, x, \varepsilon\right)=\sum_{m=-N}^{N} \varepsilon^{\gamma(m)} B_{m}(\varepsilon x) e^{i m x}+\mathcal{O}\left(\varepsilon^{(N+3) / 2}\right) .
\end{aligned}
$$

The functions $\left(A_{m}, B_{m}\right) \in\left(H_{l, u}^{N}\right)^{2}$ fulfil $\left\|\left(A_{m}, B_{m}\right)\right\|_{\left(H_{l, u}^{N}\right)^{2}} \leqq\left(C_{0}(C, N)\right.$ for a constant $C_{0}(C, N)$ independent of $\varepsilon$. The function $\gamma$ is defined by $\gamma( \pm 2)=\gamma(0)=2, \gamma( \pm 1)=1$ and $\gamma(m)=1+|m| / 2$ for $|m| \geqq 3$.

\section{The approximation property}

In this sub-section, we show that solutions of (1.1) can be approximated by the solutions of (1.4). This part follows strongly the lines of the existing literature about the validity of the Ginzburg-Landau equation. Therefore, we recommend the reading of [20] together with this sub-section.

THEOREM 2.4. Let $\varepsilon \in(0,1) \mapsto(A, B) \in C\left(\left[0, T_{0}\right],\left(H_{l, u}^{6}\right)^{2}\right)$ be a family of solutions of (1.4) which fulfils $\sup _{\varepsilon \in(0,1)} \sup _{T \in\left[0, T_{0}\right]}\|(A, B)\|_{\left(H_{1, u}^{6}\right)^{2}} \leqq C_{1}<\infty$, for a constant $C_{1}$. Then there exists $\varepsilon_{0}, C>0$, such that for all $\varepsilon \in\left(0, \varepsilon_{0}\right)$ we have solutions $(u, v)(t, x, e)$ of (1.1) with

$$
\sup _{0 \leqq t \leqq T_{0} / \varepsilon^{2}}\left\|(u, v)-\left(\psi_{u}[A, B], \psi_{v}[A, B]\right)\right\|_{\left(H_{l, u}^{4}\right)^{2}<C \varepsilon^{2} .}
$$

Proof. We use Lemma 2.1 to define so-called model filters. These operators allow us to extract certain intervals of Fourier modes from a given function. Let $\varphi$ be a $C_{0}^{\infty}$-function with the following properties: $\varphi(k) \in[0,1], \varphi(k)=1$ for $k \in[-7 / 6,-5 / 6] \cup[5 / 6,7 / 6]$, and $\varphi(k)=0$ for $k \notin[-5 / 4,-3 / 4] \cup[3 / 4,5 / 4]$. According to Lemma 2.1, we denote by $E_{c}$ the operator in physical space which is associated with the multiplier $\varphi$ in Fourier space. The operator $E_{\mathfrak{c}}$ enables us to extract the unstable Fourier modes from a function. The stable Fourier modes can be extracted with $E_{s} u=u-E_{c} u$.

The residual contains all terms which remain after inserting the ansatz $\psi_{u}, \psi_{v}$ into 
(1.1). It is defined by

$$
\begin{aligned}
& \operatorname{Res}_{1}(u, v)=-\partial_{t} u-\left(1+\partial_{x}^{2}\right)^{2} u-\alpha_{1} \partial_{x} u-\alpha_{2} \partial_{x}^{3} u+\varepsilon^{2} u+\partial_{x}\left(u^{2}+u v+v^{2}\right), \\
& \operatorname{Res}_{2}(u, v)=-\partial_{t} v-\left(1+\partial_{x}^{2}\right)^{2} v+\alpha_{1} \partial_{x} v+\alpha_{2} \partial_{x}^{3} u+\varepsilon^{2} v+\partial_{x}\left(u^{2}+u v+v^{2}\right) .
\end{aligned}
$$

Looking at the validity proofs for the Ginzburg-Landau equation in $[\mathbf{2 1}, \mathbf{2 5}]$, or especially in [20], it is obvious that Theorem 2.4 holds if the residual is sufficiently small, more precisely:

$$
\begin{aligned}
& \sup _{t \in\left[0, T_{0} / \varepsilon^{2}\right]} \sum_{j=1}^{2}\left\|E_{c} \operatorname{Res}_{j}\left(\psi_{u}, \psi_{v}\right)\right\|_{H_{l, u}^{1}} \leqq C \varepsilon^{4}, \\
& \sup _{t \in\left[0, T_{0} / \varepsilon^{2}\right]} \sum_{j=1}^{2}\left\|E_{s} \operatorname{Res}_{j}\left(\psi_{u}, \psi_{v}\right)\right\|_{H_{l, u}^{1}} \leqq C \varepsilon^{3} .
\end{aligned}
$$

It is obvious that due to the construction of the approximations $\psi_{u}$ and $\psi_{v}$ the inequality (2.4) holds, if we can estimate the terms $\partial_{t} A_{m}^{j}$ and $\partial_{t} B_{m}^{j}$ to be of order $\mathcal{O}(\varepsilon)$ for $m=0,2 ; j=0, \pm 2$. We have the same error bounds $\varepsilon^{4}$ and $\varepsilon^{3}$ as in [20], respectively, where the residual is estimated in $C_{b}^{1}$ and the solution of the GinzburgLandau equation is assumed to be in $C_{b}^{5}$, since $C_{b}^{1} \subset H_{l, u}^{1}$ and $H_{l, u}^{6} \subset C_{b}^{5}$.

The terms $\partial_{t} A_{m}^{j}$ and $\partial_{t} B_{m}^{j}$ can be expressed in terms of $\partial_{t} A$ and $\partial_{t} B$ by differentiating the algebraic equations (1.3), for instance $\left(9+2 i \alpha_{1}-8 i \alpha_{2}\right) \partial_{t} A_{2}^{0}=$ $\left.2 i\left(\mathscr{T}_{2 \alpha z t} B\right) \partial_{t} A+A\left(\mathscr{T}_{2 \alpha z t}\left(\partial_{t} B\right)+2 \alpha \varepsilon \mathscr{T}_{2 \alpha z t}\left(\partial_{X} B\right)\right)\right)$. If we can make use of $\partial_{t} A=\varepsilon^{2} \partial_{T} A$ and $\partial_{t} B=\varepsilon^{2} \partial_{T} B$, respectively, we are done. In order to do so, we need the following lemma.

Lemma 2.5. Let $(\tilde{A}, \tilde{B}) \in\left(H_{l, u}^{n}\right)^{2}$ for some $n \geqq 2$. Then there exist $C_{1}, T_{0}>0$ such that for all $\varepsilon \in(0,1)$ there are solutions $(A, B) \in C\left(\left[0, T_{0}\right],\left(H_{l, u}^{n}\right)^{2}\right) \cap C^{1}\left(\left[0, T_{0}\right],\left(H_{l, u}^{n-2}\right)^{2}\right)$ of

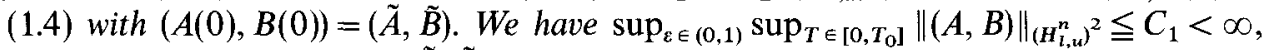
where $C_{1}$ depends only on $\|(\tilde{A}, \tilde{B})\|_{\left(H_{l, u}^{n}\right)^{2}}$. The same result holds for (1.5) for fixed $q>0$, $v \in[0,1)$, and $L=q / \varepsilon^{v}$.

Proof. From Lemma 2.1, it follows that $\left(1+4 \partial_{X}^{2}, 1+4 \partial_{X}^{2}\right)$ is the generator of an analytic semigroup $\mathscr{G}_{T}$ in $\left(L_{l, u}^{2}\right)^{2}$ with $\left\|\mathscr{G}_{T}\right\|_{\mathscr{L}\left(\left(H_{l, u}^{n}\right)^{2},\left(H_{l, u}^{m}\right)^{2}\right)} \leqq C_{m-n} \max \left(1, T^{-(m-n) / 2}\right)$. The nonlinear problem is solved by using the variation of constant formula, i.e.

$$
\left(\begin{array}{l}
A(T) \\
B(T)
\end{array}\right)=\mathscr{G}_{T}\left(\begin{array}{l}
\tilde{A} \\
\tilde{B}
\end{array}\right)+\int_{0}^{T} \mathscr{G}_{T-S}\left(\begin{array}{l}
-\left(\gamma_{1} A|A|^{2}+a_{1}+a_{2}\right)[S] \\
-\left(\gamma_{6} B|B|^{2}+b_{1}+b_{2}\right)[S]
\end{array}\right.
$$

For a sufficiently small $T_{0}$, the right-hand side defines a contraction in a ball in $C\left(\left[0, T_{0}\right],\left(H_{l, u}^{n}\right)^{2}\right)$ centred in $\mathscr{G}_{T}(\tilde{A}, \tilde{B})$. With the usual fixed-point argument, we find the required solution. The existence interval $\left[0, T_{0}\right]$ of the solutions can be chosen independently of $\varepsilon \in(0,1)$, since the Lipschitz-constant of the contraction is independent of $\varepsilon$. Its length only depends on $\|(\tilde{A}, \tilde{B})\|_{\left(H_{l, u}^{n}\right)^{2}}$. The time derivative $\left(\partial_{T} A, \partial_{T} B\right)$ is estimated by the right-hand side of $(1.4)$, which is of order $\mathcal{O}(1)$ in $H_{l, u}^{n-2}$. In the same way the result follows for (1.5).

Now we can complete the proof of Theorem 2.4. In the following, we use the abbreviations $Z=\left(H_{l, u}^{4}\right)^{2}$ and $Y=\left(H_{l, u}^{1}\right)^{2}$. We write $(1.1)$ as

$$
\partial_{t} \mathscr{U}=\Lambda \mathscr{U}+B(\mathscr{U}, \mathscr{U})
$$


where $\mathscr{U}=(u, v), \Lambda=\left(-\left(1+\partial_{x}^{2}\right)^{2}+\varepsilon^{2}-\alpha_{1} \partial_{x}-\alpha_{2} \partial_{x}^{3},-\left(1+\partial_{x}^{2}\right)^{2}+\varepsilon^{2}+\alpha_{2} \partial_{x}+\alpha_{2} \partial_{x}^{3}\right)$. The nonlinear terms are written as a symmetric bilinear mapping $B(\mathscr{U}, \mathscr{U})$ which fulfils $\|B(\mathscr{U}, \mathscr{V})\|_{Y} \leqq C\|\mathscr{U}\|_{Z}\|\mathscr{V}\|_{Z}$. The modefilters are denoted by $\mathscr{E}_{c}=\left(E_{c}, E_{c}\right)$ and $\mathscr{E}_{s}=\left(E_{s}, E_{s}\right)$, and the approximation is denoted by $\Psi=\left(\psi_{u}, \psi_{v}\right)$. Moreover, we define the critical part $\varepsilon \Psi_{c}=\mathscr{E}_{c} \Psi$ and the noncritical part $\varepsilon^{2} \Psi_{s}=\mathscr{E}_{s} \Psi$ of the approximation. We have the estimate

$$
\sup _{t \in\left[0, T_{0} / \varepsilon^{2}\right]}\left(\left\|\Psi_{c}\right\|_{Z}+\left\|\Psi_{s}\right\|_{Z}\right) \leqq C_{\psi}=\mathcal{O}(1)
$$

for $\varepsilon \rightarrow 0$. The operator $\Lambda$ generates an analytic semigroup $e^{\Lambda t}$ which can be estimated by $\left\|e^{\Lambda t} \mathscr{E}_{c}\right\|_{\mathscr{L}(Y, Z)} \leqq C e^{C \varepsilon^{2} t}$ and by $\left\|e^{\Lambda t} \mathscr{E}_{s}\right\|_{\mathscr{L}(Y, Z)} \leqq C \max \left(1, t^{-3 / 4}\right) e^{-\sigma t}$ for a $\sigma=\mathcal{O}(1)>0$. We make the ansatz $\mathscr{U}=\varepsilon \Psi_{c}+\varepsilon^{2} \Psi_{s}+\varepsilon^{2} \mathscr{R}_{c}+\varepsilon^{3} \mathscr{R}_{s}$ and define the error functions $\mathscr{R}_{c}$ and $\mathscr{R}_{s}$ to be the solutions of

$$
\begin{aligned}
\hat{o}_{t} \mathscr{R}_{c} & =\Lambda \mathscr{R}_{c}+\varepsilon^{2} L_{c}(\mathscr{R})+\varepsilon^{3} N_{c}(\mathscr{R})+\varepsilon^{-2} \mathscr{E}_{c} \operatorname{Res}(\Psi), \\
\partial_{t} \mathscr{R}_{s} & =\Lambda \mathscr{R}_{s}+L_{s}\left(\mathscr{R}_{c}\right)+\varepsilon N_{s}(\mathscr{R})+\varepsilon^{-3} \mathscr{E}_{s} \operatorname{Res}(\Psi) .
\end{aligned}
$$

We have used the abbreviations

$$
\begin{aligned}
L_{c}(\mathscr{R}) & =\mathscr{E}_{c}\left[2 B\left(\mathscr{R}_{c}, \Psi_{s}\right)+2 B\left(\mathscr{R}_{s}, \Psi_{c}\right)\right], \\
L_{s}\left(\mathscr{R}_{c}\right) & =\mathscr{E}_{s}\left[2 B\left(\mathscr{R}_{c}, \Psi_{c}\right)\right], \\
N_{c}(\mathscr{R}) & =\mathscr{E}_{c}\left[B\left(\mathscr{R}_{s}, 2 \mathscr{R}_{c}+2 \Psi_{s}+\varepsilon \mathscr{R}_{s}\right)\right], \\
N_{s}(\mathscr{R}) & =\mathscr{E}_{s}\left[2 B\left(\mathscr{R}_{c}, \Psi_{s}\right)+2 B\left(\mathscr{R}_{s}, \Psi_{c}\right)+B\left(\mathscr{R}_{c}, \mathscr{R}_{c}\right)+\varepsilon B\left(\mathscr{R}_{s}, 2 \mathscr{R}_{c}+2 \Psi_{s}+\varepsilon \mathscr{R}_{s}\right)\right] .
\end{aligned}
$$

Now system (2.7) has exactly the same properties as the corresponding systems $[20,(17)]$ and $[22,(12)]$. Following the remaining arguments of these papers, we obtain

$$
\sup _{t \in\left[0, T_{0} / \varepsilon^{2}\right]}\left(\left\|\mathscr{R}_{c}\right\|_{Z}+\left\|\mathscr{R}_{s}\right\|_{Z}\right) \leqq C=\mathscr{O}(1)<\infty
$$

for $\varepsilon \rightarrow 0$. This concludes the proof of Theorem 2.4.

\section{Mean-field coupled description}

In this section, we prove the validity of Theorem 1.2. In order to do so, it remains to show that the solutions of (1.4) can be approximated by the solutions of (1.5).

THEOREM 3.1. For arbitrary, but fixed, $q>0$ and $v \in[0,1)$ the following holds: let $\varepsilon \in(0,1) \mapsto(A, B) \in C\left(\left[0, T_{0}\right],\left(H_{l, u}^{8}\right)^{2}\right)$ be a family of solutions of $(1.5)$ to $L=q / \varepsilon^{v}$ which fulfils $\sup _{\mathrm{s} \in(0,1)} \sup _{T \in\left[0, T_{0}\right]}\|(A, B)\|_{\left(H_{l, U}^{\mathrm{s}}\right)^{2}} \leqq C_{1}<\infty$, for a constant $C_{1}$. Then there exist $\varepsilon_{0}, C>0$, such that for all $\varepsilon \in\left(0, \varepsilon_{0}\right)$ we have solutions $(\hat{A}, \hat{B})$ of $(1.4)$ with

$$
\sup _{0 \leqq t \leqq T_{0}}\|(\hat{A}, \hat{B})-(A, B)\|_{\left(H_{1, u}^{6}\right)^{2}} \leqq C \varepsilon^{1-v} .
$$

Proof of Theorem 1.2. Let $Z=\left(H_{l, u}^{4}\right)^{2}$. Using Theorem 2.4 and Theorem 3.1, we obtain

$$
\begin{aligned}
\left\|(u, v)-\left(\psi_{u}[A, B], \psi_{v}[A, B]\right)\right\|_{Z} \leqq & \left\|(u, v)-\left(\psi_{u}[\hat{A}, \hat{B}], \psi_{v}[\hat{A}, \hat{B}]\right)\right\|_{Z} \\
& +\left\|\left(\psi_{u}[\hat{A}, \hat{B}], \psi_{v}[\hat{A}, \hat{B}]\right)-\left(\psi_{u}[A, B], \psi_{v}[A, B]\right)\right\|_{Z} \\
\leqq & C \varepsilon^{2}+C \varepsilon^{2-v} .
\end{aligned}
$$


Proof of Theorem 3.1. Now let $Z=\left(H_{l, u}^{6}\right)^{2}$. The nonlinearity in (1.4) is written as a symmetric trilinear mapping $\mathcal{N}$. We have the estimate $\|\mathcal{N}(u, v, w)\|_{Z} \leqq$ $C\|u\|_{Z}\|v\|_{Z}\|w\|_{Z}$ for a $C>0$. The terms which remain after inserting a solution $\mathscr{A}=$ $(A, B)$ of (1.5) into (1.4) define again the residual $\operatorname{RES}(\mathscr{A})$ by

$$
\left(\begin{array}{l}
\operatorname{RES}_{1}(\mathscr{A}) \\
\operatorname{RES}_{2}(\mathscr{A})
\end{array}\right)=\left(\begin{array}{l}
-\partial_{T} A+A+4 \partial_{X}^{2} A-\left(\gamma_{1} A|A|^{2}+a_{1}+a_{2}\right) \\
-\partial_{T} B+B+4 \partial_{X}^{2} B-\left(\gamma_{6} B|B|^{2}+b_{1}+b_{2}\right)
\end{array}\right) .
$$

A solution $(\hat{A}, \hat{B})$ of $(1.4)$ is a sum of an approximation $\mathscr{A}=(A, B)$ and an error $\varepsilon^{\mu} \mathscr{R}$ with $\mu+\nu=1$. The error fulfils the differential equation

$$
\partial_{T} \mathscr{R}=\mathscr{L} \mathscr{R}+3 \mathscr{N}(\mathscr{A}, \mathscr{A}, \mathscr{R})+3 \varepsilon^{\mu} \mathscr{N}(\mathscr{A}, \mathscr{R}, \mathscr{R})+\varepsilon^{2 \mu} \mathscr{N}(\mathscr{R}, \mathscr{R}, \mathscr{R})+\varepsilon^{-\mu} \operatorname{RES}(\mathscr{A})
$$

with zero-initial conditions and $\mathscr{L}=\left(1+4 \partial_{X}^{2}, 1+4 \partial_{X}^{2}\right)$. The local existence and uniqueness of solutions in $Z$ follows immediately from Lemma 2.5. In order to show the assertion of Theorem 3.1, it remains to demonstrate the $\mathscr{O}(1)$-boundedness of $\mathscr{R}$ on the time interval $\left[0, T_{0}\right]$. The semigroup $\mathscr{G}_{T}=\left(S_{T}, S_{T}\right)$ generated by $\mathscr{L}$ can be estimated with the help of Lemma 2.1. This gives the existence of a $C_{2}>0$ such that $\left\|\mathscr{G}_{T}\right\|_{\mathscr{L}(Z, Z)} \leqq C_{2}$ for all $T \in\left[0, T_{0}\right]$. The application of $\mathscr{G}_{T}$ on (1.4) gives

$$
\begin{aligned}
\mathscr{R}(T)= & \int_{0}^{T} \mathscr{G}_{T-\tau}\left(3 \mathscr{N}(\mathscr{A}, \mathscr{A}, \mathscr{R})+3 \varepsilon^{\mu} \mathscr{N}(\mathscr{A}, \mathscr{R}, \mathscr{R})+\varepsilon^{2 \mu} \mathscr{N}(\mathscr{R}, \mathscr{R}, \mathscr{R})\right)(\tau) d \tau \\
& +\int_{0}^{T} \varepsilon^{-\mu} \mathscr{G}_{T-\tau} \operatorname{RES}(\mathscr{A})(\tau) d \tau .
\end{aligned}
$$

By assumption there exists a $C_{1}>0$ such that $\sup _{T \in\left[0, T_{0}\right]}\|\mathscr{A}\|_{Z} \leqq C_{1}$. Moreover, we have:

Lemma 3.2. There exist $\varepsilon_{0}, C_{\mathrm{RES}}>0$ such that for all $\varepsilon \in\left(0, \varepsilon_{0}\right)$ :

$$
\sup _{T \in\left[0, T_{0}\right]}\left\|\int_{0}^{T} \varepsilon^{-\mu \mathscr{G}_{T-\tau}} \operatorname{RES}(\mathscr{A})(\tau) d \tau\right\|_{Z} \leqq C_{\mathrm{RES}} .
$$

For the proof, see below. We show that there exist $\varepsilon_{0}, D>0$ with $\sup _{T \in\left[0, T_{0}\right]}\|\mathscr{R}(T)\|_{Z} \leqq D$ for all $\varepsilon \in\left(0, \varepsilon_{0}\right)$. Assume now the existence of such a $D$; then

$$
\|\mathscr{R}(T)\|_{Z} \leqq\left(\int_{0}^{T} C_{2} C C_{1}^{2}\|\mathscr{R}(\tau)\|_{Z} d \tau\right)+T_{0} C_{2} C\left(\varepsilon^{\mu} C_{1} D^{2}+\varepsilon^{2 \mu} D^{3}\right)+C_{\mathrm{RES}}
$$

Suppose now that

$$
\left(\varepsilon^{\mu} C_{1} D^{2}+\varepsilon^{2 \mu} D^{3}\right) \leqq 1 .
$$

Then Gronwall's inequality yields

$$
\|\mathscr{R}(T)\|_{Z} \leqq\left(T_{0} C_{2} C+C_{\mathrm{RES}}\right) e^{T_{0} C_{2} C C_{2}^{2}}=: D
$$

for $T \in\left[0, T_{0}\right]$. This defines $D$. We are done, since we can choose $\varepsilon_{0}>0$ such that the assumption (3.1) is fulfilled for all $\varepsilon \in\left(0, \varepsilon_{0}\right)$.

Proof of Lemma 3.2. Obviously we can restrict the analysis to the first component of the residual $\left(\operatorname{RES}_{1}(\mathscr{A})\right)_{1}=s_{1}+a_{2}$, where $s_{1}=a_{1}-\gamma_{5} A\left\langle\left|\mathscr{T}_{2 \alpha T / \varepsilon} B\right|^{2}\right\rangle_{L}$. The terms 
in $a_{2}$ are highly oscillating for $\varepsilon \rightarrow 0$. They can be estimated by partial integration in the following way:

$$
\begin{aligned}
& \sup _{T \in\left[0, T_{0}\right]}\left\|\int_{0}^{T} \varepsilon^{-\mu} S_{T-\tau}\left(A\left(\mathscr{T}_{2 \alpha \tau / \varepsilon} \bar{B}\right) A\right)(\tau) e^{-2 i \beta \tau / \varepsilon^{2}} d \tau\right\|_{H_{l, u}^{6}} \\
\leqq & \sup _{T \in\left[0, T_{0}\right]}\left\|\int_{0}^{T} \varepsilon^{-\mu} \partial_{\tau}\left[S_{T-\tau}\left(A\left(\mathscr{T}_{2 \alpha \tau / \varepsilon} \bar{B}\right) A\right)\right] e^{-2 i \beta \tau / \varepsilon^{2}} \varepsilon^{2} /(-2 i \beta) d \tau\right\|_{H_{l, u}^{6}} \\
& +\sup _{T \in\left[0, T_{0}\right]}\left\|\varepsilon^{-\mu}\left[S_{T-\tau}\left(A\left(\mathscr{T}_{2 \alpha \tau / \varepsilon} \bar{B}\right) A\right)\right] e^{-2 i \beta \tau / \varepsilon^{2}} \varepsilon^{2} /\left.(-2 i \beta)\right|_{\tau=0} ^{T}\right\|_{H_{l, u}^{6}} \\
\leqq & \varepsilon^{1-\mu} C\|A\|_{H_{l, u}^{8}}^{2}\|\bar{B}\|_{H_{l, u}^{8}}+\varepsilon^{2-\mu} C\left\|A_{1}\right\|_{H_{l, u}^{8}}^{2}\|\bar{B}\|_{H_{l, u}^{8}}=\mathcal{O}\left(\varepsilon^{1-\mu}\right) .
\end{aligned}
$$

We have used that $\partial_{\tau}\left(\mathscr{T}_{\alpha \tau / \varepsilon} \bar{B}\right)=\mathcal{O}(1 / \varepsilon), \partial_{\tau} S_{T-\tau}=-S_{T-\tau}\left(1+4 \partial_{X}^{2}\right)$ and that $\partial_{\tau} A$ can be expressed by the right-hand side of (1.5). All other terms in $a_{2}$ can be estimated in the same way.

It remains to bound the term

$$
\sup _{T \in\left[0, T_{0}\right]}\left\|\int_{0}^{T} \varepsilon^{-\mu} S_{T-\tau}\left[A\left(\mathscr{T}_{2 \alpha \tau / \varepsilon} \bar{B}\right)\left(\mathscr{T}_{2 \alpha \tau / \varepsilon} B\right)-A\left\langle\left|\mathscr{T}_{2 \alpha \tau / \varepsilon} B\right|^{2}\right\rangle_{L}\right] d \tau\right\|_{H_{l, u}^{6}},
$$

for $L=q \varepsilon^{-v}$. According to Lemma 2.1 the translation operator $m_{\tau}=\mathscr{T}_{2 \pi \tau / \varepsilon}$ can be represented by the multiplier $\hat{m}_{\tau}=e^{2 i \alpha k \tau / \varepsilon}$ in Fourier space. We use partial integration to estimate the term (3.2). Integrating the multiplier $m_{\mathfrak{t}}$ with respect to $\tau$ gives

$$
\begin{aligned}
& \sup _{T \in\left[0, T_{0}\right]}\left\|\int_{0}^{T} \varepsilon^{-\mu} S_{T-\tau}\left(A\left(m_{\tau}\left[\bar{B} B-\left\langle|B|^{2}\right\rangle_{L}\right]\right)\right) d \tau\right\|_{H_{l, u}^{6}} \\
& \leqq \\
& \sup _{T \in\left[0, T_{0}\right]}\left\|\int_{0}^{T} \varepsilon^{-\mu} S_{T-\tau}\left(1+4 \hat{\partial}_{X}^{2}\right)\left(A\left(m_{I} m_{\tau}\left[\bar{B} B-\left\langle|B|^{2}\right\rangle_{L}\right]\right)\right) d \tau\right\|_{H_{l, u}^{6}} \\
& \quad+\sup _{T \in\left[0, T_{0}\right]}\left\|\int_{0}^{T} \varepsilon^{-\mu} S_{T-\tau}\left(\left[\partial_{\tau} A\right]\left(m_{I} m_{\tau}\left[\bar{B} B-\left\langle|B|^{2}\right\rangle_{L}\right]\right)\right) d \tau\right\|_{H_{l, u}^{6}} \\
& \quad+\sup _{T \in\left[0, T_{0}\right]}\left\|\int_{0}^{T} \varepsilon^{-\mu} S_{T-\tau}\left(A\left(m_{I} m_{\tau} \partial_{\tau}\left[\bar{B} B-\left\langle|B|^{2}\right\rangle_{L}\right]\right)\right) d \tau\right\|_{H_{l, u}^{6}} \\
& \quad+\sup _{T \in\left[0, T_{0}\right]}\left\|\left.\varepsilon^{-\mu} S_{T-\tau}\left(A\left(m_{I} m_{\tau}\left[\bar{B} B-\left\langle|B|^{2}\right\rangle_{L}\right]\right)\right)\right|_{\tau=0} ^{T}\right\|_{H_{l, u}^{6}},
\end{aligned}
$$

with the new multiplier $\hat{m}_{I}=\varepsilon /(2 i \alpha k)$. This partial integration is well defined due to Remark 2.2 if $m_{\tau}$ is applied to a gradient of a bounded function. Since

$$
f(X)=\bar{B}(X) B(X)-\left\langle|B(X)|^{2}\right\rangle_{L} \in H_{l, u}^{8} \subset C_{b}^{1}
$$

possesses a zero in $[X-L, X+L]$, there exists a function $F \in H_{l, u}^{9}$ with $f=\partial_{X} F(X)$ and $\sup _{T \in\left[0, T_{0}\right]}\|F\|_{H_{l, u}^{9}} \leqq C \varepsilon^{-v}$. We also have $\sup _{T \in\left[0, T_{0}\right]}\left\|\partial_{T} F\right\|_{H_{l, u}^{7}} \leqq C \varepsilon^{-v}$ since the same is true for $\partial_{T} f \in H^{6}$.

The differential operator $\partial_{X}$ can be represented as a multiplier which is given in Fourier space by $i k$. Thus, according to Remark 2.2, we have $\left\|m_{I} \partial_{X}\right\|_{\mathscr{L}\left(H_{l, u}^{8}, H_{l, u}^{8}\right)}^{8}=\mathcal{O}(\varepsilon)$. We obtain $\left\|m_{\tau}\right\|_{\mathscr{L}\left(H_{l, \mu}^{n}, H_{l, \omega}^{n}\right)}=1$ since $m_{\tau}$ is a translation 
operator. Using that all scalar multipliers commute, we finally come to

$$
\left\|m_{\tau} m_{I}\left[\bar{B} B-\left\langle|B|^{2}\right\rangle_{L}\right]\right\|_{H_{l, u}^{n}} \leqq\left\|m_{\tau}\right\|_{\mathscr{L}\left(H_{l, u}^{n}, H_{l, \mu}^{n}\right)}\left\|m_{I} \partial_{X}\right\|_{\mathscr{L}\left(H_{l, \mu}^{n}, H_{l, u}^{n}\right)}\|F\|_{H_{l, \mu}^{9}} \leqq \mathcal{O}\left(\varepsilon^{1-v}\right)
$$

for all $n \in\{0, \ldots, 9\}$. From $v+\mu=1$ it follows that the last four lines of (3.3) can be estimated by $T_{0} C\|A\|_{H_{i, u}^{8}}\|F\|_{H_{i, u}^{9}}=\mathcal{O}(1)$. Therefore the proof is complete.

\section{Acknowledgment}

The author is grateful for discussions with G. Dangelmayr, W. Eckhaus, E. Knobloch and A. van Harten. Moreover, he would like to thank the referee for many helpful comments. This research was partially supported by the Deutsche Forschungsgemeinschaft DFG under grant Mi 459/2-1.

\section{References}

1 P. Bollerman. On the validity of amplitude equations. (Thesis, University of Utrecht, 1996).

2 P. Bollerman, A. van Harten and G. Schneider. On the justification of the Ginzburg-Landau approximation. In Nonlinear Dynamics and Pattern Formation in the Natural Environment, eds A. Doelman and A. van Harten, 20-36 (Harlow: Longman, 1995).

3 P. Chossat and G. Iooss. The Taylor-Couette Problem, Applied Mathematical Sciences 102 (New York: Springer, 1994).

4 P. Collet and J.-P. Eckmann. The time dependent amplitude equation for the Swift-Hohenberg problem. Comm. Math. Phys. 132 (1990), 139-53.

5 P. Coullet, S. Fauve and E. Tirapegui. Large-scale instability of nonlinear standing waves. J. Phys. Lett. 46 (1985), L787-L791.

6 G. Dangelmayr. Ginzburg-Landau description of waves in extended systems (Preprint, Universität Tübingen, 1993); in Dynamics of Nonlinear Waves in Dissipative Systems: Reduction, Bifurcation, and Stability, Pitman Research Notes in Mathematics Series (Harlow: Longman, 1996).

7 W. Eckhaus. The Ginzburg-Landau equation is an attractor. J. Nonlinear Sci. 3 (1993), 329-48.

8 W. Eckhaus and A. Shepeleva. Attraction properties of the Ginzburg-Landau manifold (Preprint 878, University of Utrecht, 1994).

9 D. Henry. Geometric theory of semilinear parabolic equations, Lecture Notes in Mathematics 840 (Berlin: Springer, 1981).

10 P. Kirrmann, G. Schneider and A. Mielke, The validity of modulation equations for extended systems with cubic nonlinearities. Proc. Roy. Soc. Edinburgh Sect. A 122 (1992), 85-91.

11 E. Knobloch. Nonlocal amplitude equations. In Pattern Formation in Complex Dissipative Systems, eds S. Kai, 263-74 (Singapore: World Scientific, 1992).

12 E. Knobloch and J. DeLuca. Amplitude equations for travelling wave correction. Nonlinearity 3 (1990), 975-80.

13 E. Knobloch and J. D. Gibbon. Coupled NLS equations for counter propagating waves in systems with reflectional symmetry. Phys. Lett. A 154 (1991), 353-6.

14 B. J. Matkowsky and V. Volpert. Coupled Ginzburg-Landau equations in gasless combustion. Phys. D 54 (1992), 203-19.

15 A. Mielke and G. Schneider. Attractors for modulation equations on unbounded domains-existence and comparison. Nonlinearity 8 (1995), 743-68.

16 A. Newell and $\mathbf{J}$. Whitehead. Finite bandwidth, finite amplitude convection. J. Fluid Mech. 38 (1969), 279-303.

17 C. A. Pereira and J. M. Vega. On the pulsating instability of two-dimensional flames. European J. Appl. Math. 3 (1992), 55-73.

18 R. D. Pierce and E. Knobloch. On the modulational stability of traveling and standing waves. Phys. Fluids 6 (1994), 1177-90.

19 R. D. Pierce and C. E. Wayne. On the validity of mean-field amplitude equations for counterpropagating wavetrains. Nonlinearity 8 (1995), 769-79.

20 G. Schneider. A new estimate for the Ginzburg-Landau approximation on the real axis. J. Nonlinear Sci. 4 (1994), 23-34.

21 G. Schneider. Error estimates for the Ginzburg-Landau approximation. J. Appl. Math. Phys. (ZAMP) 45 (1994), 433-57. 
22 G. Schneider. Global existence via Ginzburg-Landau formalism and pseudo-orbits of GinzburgLandau approximations. Comm. Math. Phys. 164 (1994), 157-79.

23 G. Schneider. Analyticity of Ginzburg-Landau modes. J. Differential Equations 121 (1995), 233-57.

24 G. Schneider. The long wave limit for a Boussinesq equation (IfAM Preprint, Universität Hannover, 1995).

25 A. van Harten. On the validity of Ginzburg-Landau's equation. J. Nonlinear Sci. 1 (1991), 397-422.

26 J. M. Vega. On the amplitude equations arising at the onset of the oscillatory instability. SIAM J. Math. Anal. 24 (1993), 603-17.

(Issued 17 June 1997) 